BULL. AUSTRAL. MATH. SOC.

VOL. $30(1984), 435-448$.

\title{
EXPONENTIAL DICHOTOMY OF \\ STRONGLY DISCONTINUOUS SEMIGROUPS
}

\author{
P. Preda and M. Megan
}

\begin{abstract}
In this paper we give necessary and sufficient conditions for exponential dichotomy of a general class of strongly continuous semigroups of operators defined on a Banach space. As a particular case we obtain a Datko theorem for exponential stability of a strongly continuous semigroup of class $C_{0}$ defined on a Banach space.
\end{abstract}

\section{Introduction}

Let $X$ be a real or complex Banach space. The norm on $X$ and on the space $L(X)$ of all bounded linear operators from $X$ into itself will be denoted by $\|\cdot\| . T(t)$ will stand for a semigroup of linear operators on $X$ which is of class $C_{0}$; that is, $T(t)$ is strongly continuous on $\mathbf{R}_{+}=[0, \infty)$ and $T(0) x=x$ for all $x$ in $X$.

Throughout in this paper we suppose that the set

$$
X_{1}=\left\{x \in X: T(\cdot) x \in L^{\infty}(X)\right\}
$$

is a closed complemented subspace of $X$. Here $L^{\infty}(X)$ denotes the Banach space of $X$-valued functions $f$ almost defined on $R_{+}$, such that $f$ is strongly measurable and essentially bounded. If $X$ is a complementary

Received 19 June 1984.

Copyright Clearance Centre, Inc. Serial-fee code: 0004-9727/84 $\$$ A2.00 + 0.00 . 
subspace of $X_{1}$ then we denote by $P_{1}$ a projection along $X_{2}$ (that is, $\left.P_{1} \in L(X), P_{1}^{2}=P_{1}, \operatorname{Ker} P_{1}=X_{2}\right)$ and by $P_{2}=I-P_{1}$ a projection along $x_{1}$.

We also shall denote

$$
T_{1}(t)=T(t) P_{1} \text { and } T_{2}(t)=T(t) P_{2} .
$$

DEFINITION 1.1. The $C_{0}$ semigroup $T(t)$ is said to be

(i) exponentially stable if and only if there are $N, \nu>0$ such that

$$
\|T(t)\| \leq N e^{-v t} \text { for all } t \geq 0 ;
$$

(ii) exponentially dichotomic if and only if there exist $N_{1}, N_{2}, v>0$ such that

$$
\left\|T_{1}(t) x\right\| \leq N_{1} e^{-\nu\left(t-t_{0}\right)}\left\|T_{1}\left(t_{0}\right) x\right\|
$$

and

$$
\begin{gathered}
\left\|T_{2}(t) x\right\| \geq N_{2} e^{\nu\left(t-t_{0}\right)}\left\|T_{2}\left(t_{0}\right) x\right\| \\
\text { for all } t \geq t_{0} \geq 0 \text { and } x \in X .
\end{gathered}
$$

Clearly, if $T(t)$ is exponentially dichotomic and $X_{1}=X$ (that is, $P_{2}=0$ ) then $T(t)$ is exponentially stable. In this case is well known the following theorem due to Datko (see [5] and [6]).

THEOREM 1.1. A necessary and sufficient condition that a strongly continuous semigroup $T(t)$ of class $C_{0}$ defined on a Banach space $X$ be exponentially stable is that for some $p \in[1, \infty)$ the integral

$$
\int_{0}^{\infty}\|T(t) x\|^{p} d t<\infty \text { for all } x \in X .
$$

In this note the above result is extended in a natural manner to the general class of exponentially dichotomic $C_{0}$ semigroups of linear 
operators defined on a Banach space $X$.

The case $T(t)=\exp (A t)$, where $A$ is a bounded linear operator has been considered in [2], [3], [4], [7] and [10]. The problem of exponential dichotony of $C_{0}$-semigroup on Banach spaces has also been studied in [9], [11] and [12].

\section{Preliminary results}

The following simple lemmas will be needed in the sequel in proving the main results.

LEMMA 2.1. Let $f, g: \mathbb{R}_{+} \rightarrow \mathbb{R}_{+}$be two continuous finctions.

(i) If

(2.1) $\inf _{t \geq 0} g(t)<1$ and $f(t) \leq g\left(t-t_{0}\right) f\left(t_{0}\right)$ for all $t \geq t_{0} \geq 0$ then there are $N, v>0$ such that

$$
f(t) \leq N e^{-\nu\left(t-t_{0}\right)} f\left(t_{0}\right) \text { for all } t \geq t_{0} \geq 0 \text {. }
$$

(ii) If

(2.3) $\sup _{t \geq 0} g(t)>1$ and $f(t) \geq g\left(t-t_{0}\right) f\left(t_{0}\right)$ for every $t \geq t_{0} \geq 0$, then there exist $N, \nu>0$ such that

$$
f(t) \geq N e^{\nu\left(t-t_{0}\right)} f\left(t_{0}\right) \text { for alz } t \geq t_{0} \geq 0 \text {. }
$$

Proof. See [7].

In the sequel for $p \in[1, \infty)$ we denote by

$$
p^{\prime}= \begin{cases}\infty & \text { if } p=1, \\ p /(p-1) & , \text { if } p>1 .\end{cases}
$$

LEMMA 2.2. For every $a>0$ there exists $b>0$ such that

$$
e^{a t^{1 / p}} \geq b t^{1 / p^{\prime}} \text { for } a z z \quad t \geq 0 \text {. }
$$

Proof. It is easy to see that for 


$$
b=\left(\alpha p^{\prime} / p\right)^{p / p^{\prime}}
$$

the above inequality holds.

LEMMA 2.3. Let $\Delta=\left\{\left(t, t_{0}\right) \in \mathbb{R}_{+}^{2}: t \geq t_{0}\right\}, p \in[1, \infty)$ and let $f: \Delta \rightarrow R_{+}$be a continuous function with the property that there exist c, $\alpha>0$ such that

$$
\int_{t_{0}}^{t} f\left(s, t_{0}\right) d s \leq c\left(t-t_{0}\right)^{1 / p^{\prime}} f\left(t, t_{0}\right)
$$

and

$$
\int_{t}^{t+1} f(u, t) d u \geq \alpha
$$

for alz $t \geq t_{0}$. Then there are $N, v>0$ such that

$$
f\left(t, t_{0}\right) \geq N e^{v\left(t-t_{0}\right)^{I / p}} \text { for every } t \geq t_{0}+1 \text {. }
$$

Proof. If we denote by

(2.10) $g\left(t, t_{0}\right)=\int_{t_{0}+1}^{t} f\left(s, t_{0}\right) d s$ and $h\left(t, t_{0}\right)=\frac{p}{c} \cdot\left(t-t_{0}\right)^{1 / p}$

then from the inequalities (2.7) and (2.8) we obtain

$$
\alpha+g\left(t, t_{0}\right) \leq c\left(t-t_{0}\right)^{1 / p^{\prime}} \frac{\partial g\left(t, t_{0}\right)}{\partial t}
$$

which implies

$$
\frac{\partial}{\partial t}\left(-\alpha e^{-h\left(t, t_{0}\right)}\right) \leq \frac{\partial}{\partial t}\left(g\left(t, t_{0}\right) e^{-h\left(t, t_{0}\right)}\right) .
$$

By integration on $\left[t_{0}+1, t\right]$ it follows that

$$
\alpha e^{-p / c}-\alpha e^{-h\left(t, t_{0}\right)} \leq g\left(t, t_{0}\right) e^{-h\left(t, t_{0}\right)}
$$

and hence using the inequality (2.11) we obtain 
(2.14) $\alpha e^{-p / c_{e}^{h\left(t, t_{0}\right)}} \leq \alpha+g\left(t, t_{0}\right) \leq c\left(t-t_{0}\right)^{1 / p^{\prime}} \cdot f\left(t, t_{0}\right)$.

From Lemma 2.2 and the preceding relation it follows that there exists $N>0$ (independent of $t$ and $t_{0}$ ) such that

$$
f\left(t, t_{0}\right) \geq N e^{h\left(t, t_{0}\right) / 2} \text { for all } t \geq t_{0}+1 \text {. }
$$

The lemma is proved.

LEMMA 2.4. If $T(t)$ is a $C_{0}$ semigroup on a Banach space $X$ then there exist $M>1, \omega>0$ such that

(2.18) $\quad M^{p} e^{\omega p} \cdot \int_{t_{0}}^{t_{0}+1}\|T(t) x\|^{p} d t \geq\left\|T\left(t_{0}+1\right) x\right\|^{p}$,

and

$$
\left\|T_{2}\left(t_{0}\right) x\right\|^{-p} \leq M^{p} e^{\omega p} \cdot \int_{t_{0}}^{t_{0}+1}\left\|T_{2}(t) x\right\|^{-p} d t
$$

for alz $t_{0} \geq 0, x \in X, t \in\left[t_{0}, t_{0}+1\right]$ and $p \in[1, \infty)$.

Proof. It is well known (see, for example, [1], pp. 165-166) that there are $M>1$ and

$$
\omega \geq \inf _{t \geq 0} \frac{\ln T(t)}{t}
$$

such that (2.16) holds.

From

$$
\left\|T\left(t_{0}+1\right) x\right\| \leq\left\|T\left(t_{0}+1-t\right)\right\|\|T(t) x\| \leq M e^{\omega}\|T(t) x\|
$$

and

$$
\|T(t) x\| \leq\left\|T\left(t-t_{0}\right)\right\|\left\|T\left(t_{0}\right) x\right\| \leq M e^{\omega}\left\|T\left(t_{0}\right) x\right\|
$$


the relation (2.17) results.

The inequalities (2.18) and (2.19) follow immediately from (2.17).

LEMMA 2.5. Let $T(t)$ be a $C_{0}$ semigroup on the Banach space $X$ and let $P_{1}$ respectively $P_{2}$ be the projection along the closed complemented subspace $x_{1}$ defined by (1.1) respectively $x_{2}=x \Theta X_{1}$. Then we have that

$$
\begin{aligned}
& T_{1}(t)=P_{1} T_{1}(t) \text { for every } t \geq 0, \\
& T_{2}(t) x \neq 0 \text { for all } t \geq 0 \text { and } x \notin x_{1},
\end{aligned}
$$

and

(2.25) if $T_{1}(t) x \neq 0$ for every $t \geq 0$ then $T(t) x \neq 0$ for all

$$
t \geq 0 \text {. }
$$

Proof. For (2.23) it is sufficient to prove that the subspace $X_{I}$ is an invariant subspace for $T(t)$.

Indeed, if $x \in X_{1}$ and $t \geq 0$ then from

(2.26) $\|T(s) T(t) x\|=\|T(t+s) x\| \leq\|T(t)\|\|T(s) x\| \leq M e^{\omega t} \sup _{s \geq 0}\|T(s) x\|$

it follows that $T(t) x \in X_{1}$.

If there exist $t \geq 0$ and $x \notin X_{1}$ such that $T_{2}(t) x=0$ then from

$$
T(s) x=T_{1}(s) x \text { for all } s \geq t
$$

and $T_{1}(\cdot) x \in L^{\infty}(X)$ it follows that $x \in X_{1}$. This contradiction proves the property $(2.24)$.

The implication (2.25) is obvious from the equality

$$
x_{1} \cap x_{2}=\{0\} \text {. }
$$

\section{The main results}

We are now ready to prove the following

THEOREM 3.1. Let $T(t)$ be a strongly continuous semigroup of 
operators of class $C_{0}$ defined on the Banach space $X$. Then $T(t)$ is exponentially dichotomic if and only if there exist $c, p \geq 1$ such that

$$
\int_{0}^{t}\left\|T_{1}(t-s)\right\|^{p} d s \leq c^{p}
$$

and

$$
\int_{t}^{\infty}\left\|T_{2}(u) x\right\|^{-p} d u \leq c^{-p} \cdot\left\|T_{2}(t) x\right\|^{-p}
$$

for alz $t \geq 0$ and $x \in X$.

Proof. Necessity. We omit the simple verification (using Definition 1.1 (ii)) that if $T(t)$ is exponentially dichotomic then it satisfies the above inequalities (3.1) and (3.2).

Sufficiency. Suppose that the $C_{0}$ semigroup $T(t)$ has the properties (3.1) and (3.2).

Let $t_{0} \geq 0, \quad x \in X$ be fixed.

(i) Firstly, we suppose that

$$
T_{1}(t) x \neq 0 \text { for all } t \geq 0 \text {. }
$$

Let $f: \Delta \rightarrow R_{+}$be the function defined by

$$
f\left(t, t_{0}\right)=\frac{1}{\| T_{1}\left(t-t_{0}\right)} \|
$$

From Lemma 2.4 it follows that there exist $M, \omega>0$ such that

$$
f(u, t) \geq e^{-\omega} / M\left\|P_{1}\right\|=\alpha \text { for all } u \in[t, t+1] \text { and } t \geq t_{0} \text {. }
$$

Hence

$$
\int_{t}^{t+1} f(u, t) d u \geq \alpha,
$$

that is, the inequality (2.8) from Lemma 2.3 holds.

From Lemmas 2.4 and 2.5 , using Hölder's inequality, we have 
(3.7) $\left\|T_{1}\left(t-t_{0}\right)\right\| \int_{t_{0}}^{t} f\left(s, t_{0}\right) d s=\int_{t_{0}}^{t}\left\|T_{1}(t-s) T_{1}\left(s-t_{0}\right)\right\| f\left(s, t_{0}\right) d s$

$$
\leq \int_{t_{0}}^{t}\left\|T_{1}(t-s)\right\| d s \leq\left(\int_{t_{0}}^{t}\left\|T_{1}(t-s)\right\|^{p} d s\right)^{1 / p}\left(t-t_{0}\right)^{1 / p^{\prime}} \leq c\left(t-t_{0}\right)^{1 / p^{\prime}} \text {. }
$$

This shows that the inequality (2.7) from Lemma 2.3 is verified.

By Lemma 2.3 there are $M_{1}, \lambda_{1}>0$ such that

(3.8)

$$
\left\|T_{1}\left(t-t_{0}\right)\right\| \leq M_{1} e^{-\lambda_{1}\left(t-t_{0}\right)^{1 / p}} \text { for all } t \geq t_{0}+1 \text {. }
$$

From this inequality and

$$
\left\|T_{1}(t) x\right\| \leq\left\|T_{1}\left(t-t_{0}\right)\right\| \cdot\left\|T_{1}\left(t_{0}\right) x\right\|
$$

we obtain that there is $N>0$ such that

(3.10) $\quad\left\|T_{1}(t) x\right\| \leq N e^{-\lambda_{1}\left(t-t_{0}\right)^{I / p}}\left\|T_{1}\left(t_{0}\right) x\right\|$, for all $t \geq t_{0}$.

(ii) Suppose now that

$$
\text { there exists } s_{0}>0 \text { such that } T_{1}\left(s_{0}\right) x=0 .
$$

Then

$$
T_{1}(s) x=T_{1}\left(s-s_{0}\right) T_{1}\left(s_{0}\right) x=0 \text { for all } s \geq s_{0} \text {. }
$$

Let $t_{x}>0$ such that $T_{1}\left(t_{x}\right) x=0$ and $T_{1}(t) x \neq 0$ for every $t<t_{x}$

If $t \geq t_{0} \geq t_{x}$ or $t \geq t_{x} \geq t_{0}$ then $T_{1}(t) x=0$ and hence the inequality (3.10) holds.

If $t_{x} \geq t \geq t_{0} \geq 0$ then from the preceding case $(3.10)$ is also verified.

From Lemma 2.1 it follows that there exist $N_{1}, \nu_{1}>0$ such that

$$
\left\|T_{1}(t) x\right\| \leq N_{1} e^{-\nu_{1}\left(t-t_{0}\right)}\left\|T_{1}\left(t_{0}\right) x\right\|
$$


for all $t \geq t_{0} \geq 0$ and $x \in X$. This shows that the semigroup $T_{1}(t)$ is exponentially stable.

For $T_{2}(t)$ we consider the function $g:\left[t_{0}, \infty\right) \rightarrow \mathbb{R}_{+}$defined by

$$
g(t)=\int_{t}^{\infty}\left\|T_{2}(u) x\right\|^{-p} d u
$$

The inequality (3.2) shows that

$$
c^{p} g(t) \leq-\frac{d g(t)}{d t}
$$

and hence, by integration, we obtain

$$
g(t) \leq g\left(t_{0}\right) \cdot e^{\left(t_{0}-t\right) c^{p}} \text { for all } t \geq t_{0},
$$

which implies that

$$
g(t) e^{\left(t-t_{0}\right) c^{p}} \leq g\left(t_{0}\right) \leq e^{-p}\left\|T_{2}\left(t_{0}\right) x\right\|^{-p} .
$$

Therefore

$$
\int_{t}^{t+1}\left\|T_{2}(u) x\right\|^{-p} d u \cdot e^{\left(t-t_{0}\right) c^{p}} \leq e^{-p}\left\|T_{2}\left(t_{0}\right) x\right\|^{-p}
$$

for every $t \geq t_{0}$.

If we denote by $\alpha=M^{\omega}$ then from Lemma 2.4 it follows that

$$
\alpha^{-p_{1}}\left\|T_{2}(t) x\right\|^{-p_{e}} e^{\left(t-t_{0}\right) c^{p}} \leq c^{-p_{\| T_{2}}\left(t_{0}\right) x \|^{-p}}
$$

and hence there is $N_{2}, v_{2}>0$ such that

(3.20) $\left\|T_{2}(t) x\right\| \geq N_{2} e^{v_{2}\left(t-t_{0}\right)}\left\|T_{2}\left(t_{0}\right) x\right\|$ for all $t \geq t_{0} \geq 0$ and $x \in X$.

If $v=\min \left\{v_{1}, v_{2}\right\}$ then from (3.13) and (3.20) it follows that the inequalities (1.4) and (1.5) hold and hence $T(t)$ is exponentially dichotomic. 
THEOREM 3.2. The $C_{0}$ semigroup $T(t)$ is exponentially dichotomic if and only if there are $c, p \geq 1$ such that

$$
\int_{0}^{t}\left\|T_{1}(t-s) x\right\|^{p} d s \leq c^{p}\|x\|^{p}
$$

and

$$
\int_{t}^{\infty}\left\|T_{2}(u) x\right\|^{-p} d u \leq c^{-p}\left\|T_{2}(t) x\right\|^{-p},
$$

for alz $t \geq 0$ and $x \in X$.

Proof. Necessity is obvious from the preceding theorem.

Sufficiency. From the hypothesis (3.21) it results that

$$
\int_{0}^{\infty}\left\|T_{1}(s) x\right\|^{p} d s \leq c^{p} .\|x\|^{p} \text { for all } x \in X .
$$

From Theorem 1.1 and (2.23) it follows that $T_{1}(t)$ is an exponentially stable semigroup. Hence there is $N_{1}, v_{1}>0$ such that

(3.24) $\quad\left\|T_{1}(t) x\right\| \leq\left\|T_{1}\left(t-t_{0}\right)\right\| \cdot\left\|T_{1}\left(t_{0}\right) x\right\| \leq N_{1} e^{-\nu_{1}\left(t-t_{0}\right)}\left\|T_{1}\left(t_{0}\right) x\right\|$ for all $t \geq t_{0} \geq 0$ and $x \in X$.

Then using this inequality and the proof of the preceding theorem we obtain that $T(t)$ is exponentially dichotomic.

As a particular case (when $P_{2}=0$ ) we obtain Datko's result:

COROLLARY 3.1. Let $T(t)$ be a $c_{0}$ semigroup of Zinear operators defined on the Banach space $X$. The following statements are equivalent:

(i) $T(t)$ is exponentially stable;

(ii) there are $c, p \geq 1$ such that

$$
\int_{0}^{\infty}\|T(t)\|^{p} d t \leq c^{p}
$$

(iii) there exist $c, p \geq 1$ such that 


$$
\int_{0}^{\infty}\|T(t) x\|^{p} d t \leq e^{p} \cdot\|x\|^{p} \text { for } a z z \quad x \in X .
$$

Proof. Is obvious from Theorems 3.1 and 3.2 .

REMARK 3.1. In the proofs of Theorem 3.1 and that of the equivalence $(i) \Leftrightarrow(i i)$ from the preceding corollary we have not used Datko's theorem.

THEOREM 3.3. A necessary and sufficient condition for the $C_{0}$ semigroup $T(t)$ to be exponentially dichotomic is the existence of positive constants $m, c$ and $p \geq 1$ such that

$$
\begin{aligned}
\int_{t}^{\infty}\left\|T_{1}(u-t)\right\|^{p} d u & \leq c^{p}, \\
\left\|T_{2}(t+1) x\right\| & \geq m\left\|T_{2}(t) x\right\|,
\end{aligned}
$$

and

$$
\int_{0}^{\infty}\left\|T_{2}(s) x\right\|^{p} d s \leq c^{p}\left\|T_{2}(t) x\right\|^{p},
$$

for all $t \geq 0$ and $x \in X$.

Proof. Necessity is a simple verification.

Sufficiency. From

$$
\int_{0}^{t}\left\|T_{1}(t-s)\right\|^{p} d s=\int_{0}^{t}\left\|T_{1}(s)\right\|^{p} d s \leq \int_{t}^{\infty}\left\|T_{1}(u-t)\right\|^{p} d u \leq e^{p}
$$

and the proof of Theorem 3.1 it follows that the inequality (3.13) holds.

Let $t_{0} \geq 0$ and $x \in X$. Let now $f$ be the real function

$$
f: \mathbf{R}_{+} \rightarrow \mathbf{R}_{+}, f(t)=\int_{0}^{t}\left\|T_{2}(s) x\right\|^{p} d s .
$$

From the above inequality (3.29) we have that

$$
f(t) \leq c^{p} \cdot \frac{d f(t)}{d t}
$$

and hence by integration it follows

$$
e^{-c^{-p}} e^{\left(t-t_{0}\right) / c^{p}} f\left(t_{0}+1\right) \leq f(t) \leq c^{p} \cdot\left\|T_{2}(t) x\right\|^{p}
$$


for every $t \geq t_{0}+1$.

On the other hand from (2.18) and (3.29) it results that there exists $m>0$ such that

$$
\begin{aligned}
f\left(t_{0}+1\right) & \geq \int_{t_{0}}^{t_{0}+1}\left\|T_{2}(s) x\right\|^{p} d s \geq \alpha^{-p_{\|}}\left\|T_{2}\left(t_{0}+1\right) x\right\|^{p} \\
& \geq m^{p} \alpha^{-p_{\| T_{2}}\left(t_{0}\right)_{x} \|^{p}}, \text { where } a=M e^{\omega} .
\end{aligned}
$$

Finally, we obtain

$$
\left\|T_{2}(t) x\right\| \geq N_{3} e^{\nu_{2}\left(t-t_{0}\right)} \cdot\left\|T_{2}\left(t_{0}\right) x\right\|,
$$

for all $t \geq t_{0}+1$ and $x \in X$, where

$$
N_{3}=\frac{m}{c} \cdot e^{-c^{-p} / p} \text { and } v_{2}=\frac{1}{p c^{p}}
$$

If $t_{0} \leq t \leq t_{0}+1$ then from (2.17) and (3.35) we obtain

(3.37) $\left\|T_{2}(t) x\right\| \geq \frac{\left\|T_{2}\left(t_{0}+1\right) x\right\|}{\alpha} \geq \frac{N_{3} e^{\nu_{2}}}{\alpha}\left\|T_{2}\left(t_{0}\right) x\right\| \geq \frac{N_{3}}{\alpha} e^{\nu_{2}\left(t-t_{0}\right)} \cdot\left\|T_{2}\left(t_{0}\right) x\right\|$ and hence

(3.38) $\left\|T_{2}(t) x\right\| \geq \frac{N_{3}}{\alpha} e^{\nu_{2}\left(t-t_{0}\right)}\left\|T_{2}\left(t_{0}\right) x\right\|$ for all $t \geq t_{0} \geq 0$ and $x \in X$.

If $N_{2}=N_{3} / \alpha$ and $v=\min \left\{v_{1}, v_{2}\right\}$ then (1.4) and (1.5) are satisfied and hence $T(t)$ is exponentially dichotomic.

COROLLARY 3.2. Let $T(t)$ be a $C_{0}$ semigroup of linear operators defined on a Banach space $X$. Then $T(t)$ is exponentially dichotomic if and only if there exist $m, c>0$ and $p \geq 1$ such that

$$
\int_{t}^{\infty}\left\|T_{1}(u-t) x\right\|^{p} d u \leq c^{p} \cdot\|x\|^{p},
$$

$$
\int_{0}^{t}\left\|T_{2}(s) x\right\|^{p} d s \leq c^{p} \cdot\left\|T_{2}(t) x\right\|^{p},
$$


and

$$
\left\|T_{2}(t+1) x\right\| \geq m\left\|T_{2}(t) x\right\|,
$$

for alz $t \geq 0$ and $x \in X$.

Proof. Similar to the proof of Theorem 3.2.

\section{References}

[1] A.V. Balakrishnan, Applied fronctional analysis (Applications of Mathematics, 3. Springer-Verlag, New York, Heidelberg, Berlin, 1976).

[2] R. Conti, "On the boundedness of solutions of ordinary differential equations", Fronkcial. Ekvac. 9 (1966), 23-26.

[3] W.A. Coppe1, Dichotomies in stability theory (Lecture Notes in Mathematics, 629. Springer-Verlag, Berlin, Heidelberg, New York, 1978).

[4] J.L. Dalecki and M.G. Krein, Stability of solutions of differential equations in Banach spaces (Translations of Mathematical Monographs, 43. American Mathematical Society, Providence, Rhode Island, 1974).

[5] R. Datko, "Extending a theorem of A.M. Liapunov to Hilbert space", $J$. Math. Anal. Appl. 32 (1970), 610-616.

[6] R. Datko, "Uniform asymptotic stability of evolutionary processes in a Banach space", SIAM J. Math. Anal. 3 (1973), 428-445.

[7] José Luis Massera and Juan Jorge Schäffer, "Linear differential equations and functional analysis, I", Ann. of Math. (2) 67 (1958), 517-573.

[8] José Luis Massera, Juan Jorge Schätfer, Linear differential equations and finction spaces (Pure and Applied Mathematics, 21. Academic Press, New York and London, 1966).

[9] Mihail Megan and Petre Preda, "On exponential dichotony in Banach spaces", Bull. Austral. Math. Soc. 23 (1981), 293-306. 
[10] Kenneth J. Palmer, "Two linear system criteria for exponential dichotomy", Ann. Mat. Pura Appl. (4) 124 (1980), 199-216.

[11] P. Preda and M. Megan, "Admissibility and dichotomy for $C_{0}$-semigroups", An. Univ. Timisoara Ser. Stint. Mat. 18 (1980), $153-168$.

[12] Petre Preda and Mihail Megan, "Nonuniform dichotomy of evolutionary processes in Banach spaces", Bull. Austral. Math. Soc. 27 (1983), 31-52.

University of Timisoara,

Department of Mathematics,

Bul. V. Pârvan nr. 4,

1900 - Timisoara,

RS Romania. 